\title{
Improvement of receptor model use in analytical aspect
}

\author{
Chu-Fang Wang ${ }^{\mathrm{a}}$, Pen-Chi Chiang ${ }^{\mathrm{b}}$, Man-Ting Cheng ${ }^{\mathrm{c}}$, Hung-Lung Chiang ${ }^{\mathrm{d}, *}$ \\ ${ }^{a}$ Department of Biomedical Engineering and Environmental Science, National Tsing-Hwa University, Hsinchu 30013, Taiwan \\ ${ }^{\mathrm{b}}$ Graduate Institute of Environmental Engineering, National Taiwan University, Taipei 10617, Taiwan \\ ${ }^{\mathrm{c}}$ Department of Environmental Engineering, National Chung-Hsing University, Taichung 402, Taiwan \\ ${ }^{\mathrm{d}}$ Department of Health Risk Management, China Medical University, Taichung 40402, Taiwan
}

Received 10 April 2007; received in revised form 24 July 2007; accepted 30 July 2007

\begin{abstract}
In this study, four certified particle standards including NIST SRM 1648 urban particulate matter, BCR Reference Material No. 176 city waste incineration ash, NIST SRM 2709 San Joaquin soil, and NIST SRM 1633b coal fly ash were used to simulate ambient particulate matter. Twenty-five samples were prepared with the four certified particulate standards. A total of 23 elements were analyzed per sample, 19 by ICP-AES and ICP-MS, three by IC, and one element, $\mathrm{Si}$, by spectrophotometer. Results showed that combining the three IC-analyzed ionic species with the 19 ICP-AES/MS analyzed elements into the CMB model did not improve the source identification significantly. In addition, when all 23 analyzed chemical species per sample were used in the CMB model, they were still not good enough to effectively make the parameters of the CMB model fit the statistical criteria. Some of high variation and low recovery chemical species, i.e. Cd, $\mathrm{V}$, Sb, etc., may have caused poor CMB model simulation. Omitting some poor quality analyzed species (such as relative analysis error $>20 \%$ ) could improve the CMB model simulation. Therefore, high quality chemical species data are important for the CMB model. In addition, co-linearity of source profiles also affects the CMB model; combining the colinear sources could enhance the solubility of the CMB model. In this study, a two-step procedure was developed for CMB model simulation to improve source identification.
\end{abstract}

(C) 2007 Elsevier Ltd. All rights reserved.

Keywords: Chemical mass balance (CMB); Receptor model; Fingerprints

\section{Introduction}

Since it was first proposed in the early 1970s (Hidy and Friedlander, 1972; Kneip et al., 1972; Winchester and Nifong, 1971), the chemical mass balance $(\mathrm{CMB})$ receptor model has become one of the most successful mathematical methods used to apportion potential source contributions and to

\footnotetext{
*Corresponding author. Tel.: + 886422079685 ; fax: +886422079687.

E-mail address: hlchiang@mail.cmu.edu.tw (H.-L. Chiang).
}

develop appropriate air quality management strategies (Gordon, 1980, 1988; Hopke and Dattner, 1982, 1985; Watson, 1989; Begum et al., 2005; Kim et al., 2005; Marmur et al., 2005; Ward and Smith, 2005; Feng et al., 2006). However, one of the largest impediments to the receptor model today is the lack of accurate, precise, and comparable analytical data for determining chemical profiles ("fingerprints") of ambient and source data sets (Wang et al., 1995a, b; Robinson et al., 2006; Bhave et al., 2007).

The chemical composition of ambient air samples is by no means needed quantitatively as input for 
the $\mathrm{CMB}$ receptor model. The obtained analytical data contain not only direct information on the concentrations measured during each sampling period, but also a wealth of indirect information in the correlations that exist between the pollution sources and sampling sites (Gordon, 1980). The CMB model input data include the ambient concentrations of various species and their fractional amount in each source-type emission. However, even with the best of sampling and analytical methods, only a limited number of species are generally available for the subsequent model analysis. Thus, in a complex air shed, the resolution and precision of the model analysis may not only be limited by the number of chemical species but also by the reliability of these data on which the mathematical analysis is based. To obtain a better CMB result the uncertainty estimates of these values are required. It is therefore necessary to weigh the importance of input data values in the solution and to calculate the uncertainties of the source contributions. Currently used CMB software (Watson et al., 1990, 1998; Christensen, 2004; Christensen and Gunst, 2004) applies the effective variance solution which can provide realistic estimates of the uncertainties for the source contributions and give greater influence to chemical species with higher precision in both the source and receptor measurements than to species with lower precision.

The CMB model assumptions include constant compositions of source emissions, no reaction between chemical species, known characterized information about emission sources, linearly independent of source compositions and enough chemical information about the species. These assumptions for the CMB model are fairly restrictive and difficult to comply with in actual practice. A number of tests to determine the CMB model's abilities to tolerate deviations from these assumptions have been performed (Gordon et al., 1981; Henry, 1982; Currie et al., 1984; Dzubay et al., 1984; DeCesar et al., 1985; Javitz et al., 1988a, b; Christensen and Gunst, 2004). All these tests obtained the same conclusions that deviations from these assumptions increase the stated uncertainties of the source contribution estimates. None of them has ever quantified the influence of accurate and precise chemical information on the estimated source contributions.

This paper evaluates the influence of obtained analytical data on the solution of source contributions via the $\mathrm{CMB}$ model analysis and presents a two-step data evaluation procedure for evaluating the precision of analytical data of CMB modeling instead of optimizing analytical data. To provide sufficient information on air samples in an effort to improve the resolution of receptor model analysis, an experiment using mixed standards to simulate real samples and test the model analysis has been performed.

\section{Materials and methods}

\subsection{Selection of source profiles}

To verify the effectiveness of the CMB model from the extent of the number of chemical species and the precision and accuracy of chemical species in samples, four standard sources was selected to simulate the particulate sources in ambient air: (1) NIST SRM 1648 urban particulate matter, (2) BCR Reference Material No. 176 city waste incineration ash, (3) NIST SRM 2709 San Joaquin soil, and (4) NIST SRM 1633b coal fly ash. Among these simulated sources, waste incineration ash and coal fly ash can be categorized as industrial-type "smoke-stacks" and are considered area sources in emission inventories. On the other hand, urban particulate and soil standards represent fugitive emission sources such as wind-blown dust, motor vehicular exhaust, and other emission sources in the metropolitan.

Table 1 lists the certified concentrations of the various species in the selected standard sources which serve as input data for the CMB modeling. For chemical species whose concentration was not certified, a $5 \%$ relative standard deviation was estimated. Generally, 5\% standard deviation is a criterion for duplicate analysis of standard. Based on the certified species of standard particles, the standard deviation of most species is in the range of $1-10 \%$. When the standard deviation is less than $10 \%$, the effect on the model is insignificant and reasonable (data not shown). A total of 23 species, which can be determined in our laboratory, was used to distinguish one source from another.

\subsection{Sample preparation}

To effectively study the influence of obtained analytical data on the solution of source contributions via the CMB model analysis, 25 simulated samples (in six groups) were prepared. Table 2 lists 
Table 1

Certified concentrations (in $\mathrm{ng} \mathrm{mg}^{-1}$ ) of various chemical species in four different standards

\begin{tabular}{|c|c|c|c|c|}
\hline Chemical species & NIST SRM 1648 & BCR No. 176 & NIST SRM 2709 & NIST SRM $1633 b$ \\
\hline $\mathrm{Al}$ & $34200 \pm 1100$ & $101000 \pm 5000^{\mathrm{a}}$ & $75000 \pm 600$ & $150500 \pm 2700$ \\
\hline As & $115 \pm 10$ & $93.3 \pm 4.5^{\mathrm{a}}$ & $17.7 \pm 0.8$ & $136 \pm 2.6$ \\
\hline $\mathrm{Ba}$ & $737 \pm 37^{\mathrm{a}}$ & $3910 \pm 196^{\mathrm{a}}$ & $968 \pm 40$ & $709 \pm 27$ \\
\hline $\mathrm{Ca}$ & $5600 \pm 280^{\mathrm{a}}$ & $8800 \pm 440^{\mathrm{a}}$ & $18900 \pm 500$ & $15100 \pm 600$ \\
\hline $\mathrm{Cd}$ & $75 \pm 7$ & $412 \pm 9$ & $0.38 \pm 0.01$ & $0.784 \pm 0.006$ \\
\hline $\mathrm{Cr}$ & $403 \pm 12$ & $863 \pm 38.0$ & $130 \pm 4$ & $198.2 \pm 4.7$ \\
\hline $\mathrm{Cu}$ & $609 \pm 27$ & $1300 \pm 30$ & $34.6 \pm 0.7$ & $112.8 \pm 2.6$ \\
\hline $\mathrm{Fe}$ & $39100 \pm 1000$ & $21300 \pm 1100$ & $35000 \pm 1100$ & $77800 \pm 2300$ \\
\hline $\mathrm{K}$ & $10500 \pm 100$ & $45000 \pm 2300^{\mathrm{a}}$ & $20300 \pm 600$ & $19500 \pm 300$ \\
\hline $\mathrm{Mg}$ & $8000 \pm 400^{\mathrm{a}}$ & $21800 \pm 1100^{\mathrm{a}}$ & $15100 \pm 500$ & $4820 \pm 80$ \\
\hline $\mathrm{Mn}$ & $786 \pm 17$ & $1390 \pm 70^{\mathrm{a}}$ & $538 \pm 17$ & $132 \pm 1.7$ \\
\hline $\mathrm{Na}$ & $4250 \pm 20$ & $43000 \pm 2200^{\mathrm{a}}$ & $11600 \pm 300$ & $2010 \pm 30$ \\
\hline $\mathrm{Ni}$ & $82 \pm 3$ & $12.4 \pm 4.2$ & $88 \pm 5$ & $120.6 \pm 1.8$ \\
\hline $\mathrm{Pb}$ & $6550 \pm 80$ & $1090 \pm 17$ & $18.9 \pm 0.5$ & $68.2 \pm 1.1$ \\
\hline $\mathrm{Sb}$ & $4.50 \pm 0.23^{\mathrm{a}}$ & $412 \pm 18$ & $0.79 \pm 0.04$ & $6 \pm 0.3^{\mathrm{a}}$ \\
\hline $\mathrm{Si}$ & $110000 \pm 5000^{\mathrm{a}}$ & $140000 \pm 7000$ & $296600 \pm 2300$ & $230200 \pm 800$ \\
\hline $\mathrm{Sr}$ & $24.4 \pm 1.2^{\mathrm{a}}$ & $46.2 \pm 2.3^{\mathrm{a}}$ & $231 \pm 2$ & $1041 \pm 14$ \\
\hline $\mathrm{Ti}$ & $4000 \pm 200^{\mathrm{a}}$ & $851 \pm 43^{\mathrm{a}}$ & $3420 \pm 240$ & $7910 \pm 140$ \\
\hline $\mathrm{V}$ & $127 \pm 7$ & $10.0 \pm 0.5^{\mathrm{a}}$ & $112 \pm 5$ & $295.7 \pm 3.6$ \\
\hline $\mathrm{Zn}$ & $4760 \pm 140$ & $25800 \pm 380$ & $106 \pm 3$ & $210 \pm 10.5^{\mathrm{a}}$ \\
\hline $\mathrm{F}^{-}$ & $437 \pm 22^{\mathrm{a}}$ & $435 \pm 22^{\mathrm{a}}$ & $366 \pm 18$ & $405 \pm 20$ \\
\hline $\mathrm{Cl}^{-}$ & $198 \pm 10^{\mathrm{a}}$ & $4150 \pm 208^{a}$ & - & - \\
\hline $\mathrm{SO}_{4}^{2-}$ & $154200 \pm 700$ & $9680 \pm 480$ & $70.2 \pm 3.5$ & $475 \pm 24$ \\
\hline
\end{tabular}

${ }^{\text {a }}$ Values are not certified; a $5 \%$ relative standard derivation is estimated.

the amount of mass of the various standard sources in each sample.

In group "A", a 7:1:1:1 ratio of source contribution was designed to investigate the influence of a dominant source on the other minor sources. Group "B" was arranged in a 4:3:2:1 sequential ratio for different standard sources to test whether the apportion order would be altered by the CMB model analysis or not. To determine the capability of the CMB software in distinguishing a nonexistent source and colinearity problem, a 1:1:1:0 ratio of source contribution was prepared in group " $\mathrm{C}$ " and then tested with all four source profiles in CMB model analysis. Group "D" compared the colinearity occurring between the urban particulate matter source ( $40 \%$ in source profile) and other standard sources (in 4:4:1:1 source profile). Group "E" included three samples with an equal ratio of source contribution but different amounts of mass, which was designed to examine the influence of sample mass on the analytical result as well as model results. Finally, seven randomly prepared samples in various source contributions were tested to verify the effectiveness of the proposed two-step CMB modeling procedure.

\subsection{Chemical analysis}

During the course of this work, an $\mathrm{HNO}_{3}+$ $\mathrm{HClO}_{4}+\mathrm{HF}(3: 5: 2 \mathrm{v} / \mathrm{v})$ acid mixture closed vessel digestion method proposed in previous work (Wang et al., 1995a) was employed to completely dissolve the samples. The digested solution was then analyzed by ICP-MS. A Jarrel-Ash ICAP 9000 multi-collector type ICP-AES was employed for determining $\mathrm{Al}, \mathrm{Ca}, \mathrm{Fe}, \mathrm{K}, \mathrm{Mg}, \mathrm{Na}, \mathrm{S}$ and $\mathrm{Zn}$ while a SCIEX Elan Model 5000 ICP-MS manufactured by Perkin-Elmer was employed for determining As, $\mathrm{Ba}, \mathrm{Cd}, \mathrm{Cr}, \mathrm{Cu}, \mathrm{Mn}, \mathrm{Ni}, \mathrm{Pb}, \mathrm{Sb}, \mathrm{Sr}$ and V. The detailed experimental procedure can be found in Wang et al. (1996a, b).

For IC analysis, samples were immersed in deionized water and extracted by ultrasonic cleaner (Bransonic) for $4 \mathrm{~h}$. The filtrate was syringed into the Ion Chromatograph (DIONEX, S2000I) for ionic species measurements. Concentrations of $\mathrm{Cl}^{-}$, $\mathrm{F}^{-}$, and $\mathrm{SO}_{4}^{2-}$ were then used for receptor model analysis.

$\mathrm{Si}$ content was analyzed by spectrophotometer (Himadzu UV-2100) and the method was set up in a previous study (Wang et al., 1997). 
Table 2

Masses (mg) and relative ratios of various standard sources in each sample

\begin{tabular}{|c|c|c|c|c|c|}
\hline $\begin{array}{l}\text { Sample } \\
\text { no. }\end{array}$ & Mass & $\begin{array}{l}\text { SRM } \\
1648\end{array}$ & $\begin{array}{l}\text { BCR } \\
176\end{array}$ & $\begin{array}{l}\text { SRM } \\
2709\end{array}$ & $\begin{array}{l}\text { SRM } \\
1633 b\end{array}$ \\
\hline $\mathrm{A} 1$ & 10.0 & 0.7 & 0.1 & 0.1 & 0.1 \\
\hline A2 & 10.0 & 0.1 & 0.7 & 0.1 & 0.1 \\
\hline A3 & 10.0 & 0.1 & 0.1 & 0.7 & 0.1 \\
\hline A4 & 10.0 & 0.1 & 0.1 & 0.1 & 0.7 \\
\hline B1 & 10.0 & 0.4 & 0.3 & 0.2 & 0.1 \\
\hline B2 & 10.0 & 0.1 & 0.4 & 0.3 & 0.2 \\
\hline B3 & 10.0 & 0.2 & 0.1 & 0.4 & 0.3 \\
\hline B4 & 10.0 & 0.3 & 0.2 & 0.1 & 0.4 \\
\hline $\mathrm{Cl}$ & 10.0 & 0.33 & 0.33 & 0.33 & 0.0 \\
\hline $\mathrm{C} 2$ & 10.0 & 0.0 & 0.33 & 0.33 & 0.33 \\
\hline C3 & 10.0 & 0.33 & 0.0 & 0.33 & 0.33 \\
\hline $\mathrm{C} 4$ & 10.0 & 0.33 & 0.33 & 0.0 & 0.33 \\
\hline D1 & 10.0 & 0.4 & 0.4 & 0.1 & 0.1 \\
\hline D2 & 10.0 & 0.4 & 0.1 & 0.4 & 0.1 \\
\hline D3 & 10.0 & 0.4 & 0.1 & 0.1 & 0.4 \\
\hline E1 & 4.0 & 0.25 & 0.25 & 0.25 & 0.25 \\
\hline E2 & 8.0 & 0.25 & 0.25 & 0.25 & 0.25 \\
\hline E3 & 16.0 & 0.25 & 0.25 & 0.25 & 0.25 \\
\hline $\mathrm{F} 1$ & 14.1 & 0.38 & 0.13 & 0.18 & 0.31 \\
\hline $\mathrm{F} 2$ & 12.6 & 0.34 & 0.26 & 0.18 & 0.21 \\
\hline F3 & 12.0 & 0.13 & 0.41 & 0.28 & 0.19 \\
\hline F4 & 12.9 & 0.19 & 0.33 & 0.23 & 0.25 \\
\hline F5 & 13.5 & 0.27 & 0.25 & 0.10 & 0.38 \\
\hline F6 & 14.1 & 0.12 & 0.13 & 0.38 & 0.38 \\
\hline F7 & 11.6 & 0.26 & 0.14 & 0.34 & 0.26 \\
\hline
\end{tabular}

\section{4. $C M B$ modeling}

The CMB model (U.S. EPA standard CMB8.2 software) was used to determine the suspended particulate matter concentration $\mu \mathrm{g} \mathrm{m}^{-3}$ contributed to the stations from the potential air pollution sources (EPA, 2000). In order to obtain more accurate and precise estimation results, statistical evaluation with linear least-squares method (LLS) and effective variance techniques (EVT) including percent total mass (PTM, 80-120\%), $r$-square $\left(r^{2}\right.$ $>0.8), \chi$-square statistics $\left(\chi^{2}<4\right)$ were performed (EPA, 2000).

\section{Results and discussion}

\subsection{Validation of analytical data's quality}

During the course of this work, each simulated sample was analyzed at least three times to determine the concentration and variation of each chemical species. The obtained results (M) were compared with the certified values $(\mathrm{S})$, which are calculated based on the mixed ratios of simulated sources and certified concentrations of various species in each source profile (Tables 1 and 2).

A test of the data's quality is the comparison of measured-to-simulated ratio $(\mathrm{M} / \mathrm{S})$ for individual species. Fig. 1 shows the $\mathrm{M} / \mathrm{S}$ ratios of chemical elements for each sample. For most species, the $\mathrm{M} / \mathrm{S}$ values lie between 0.9 and 1.1 , but several are seriously at variance, notably $\mathrm{Cd}, \mathrm{As}, \mathrm{Ni}, \mathrm{V}$ and $\mathrm{Sb}$. The poor fits for these species strongly suggest that there are serious errors in the analytical results of these species.

However, interference problems due to the acidderived background ions formed during the ion extracting process of ICP-MS can significantly degrade the sensitivities of many elemental determinations, especially that of ${ }^{75} \mathrm{As}$ and ${ }^{51} \mathrm{~V}$ (Kawaguchi et al., 1987; Olivares and Houk, 1986). Previous studies (Larsen and Sturup, 1994; Sheppard et al., 1994) revealed that chloride, introduced via reagents or samples, can give rise to the formation of ${ }^{40} \mathrm{Ar}^{35} \mathrm{Cl}^{+},{ }^{37} \mathrm{Cl}^{14} \mathrm{~N}^{+}$and ${ }^{35} \mathrm{Cl}^{16} \mathrm{O}^{+}$, which may seriously inhibit the deviation of ${ }^{75} \mathrm{As}^{+}$and ${ }^{51} \mathrm{~V}^{+}$. In addition, the polyatomic interference of ${ }^{25} \mathrm{Mg}^{35} \mathrm{Cl}^{-}$, and ${ }^{23} \mathrm{Na}^{37} \mathrm{Cl}^{-}$could affect the analysis of ${ }^{60} \mathrm{Ni}$ (Hu et al., 2006). Generally, low cadmium recovery is caused by the low $\mathrm{Cd}$ content in the sample, spectral interference from other elements i.e. arsenic and iron (Lambkin and Alloway, 2000), and polyatomic ions, i.e., ${ }^{95} \mathrm{Mo}^{16} \mathrm{O}^{-},{ }^{94} \mathrm{Zr}^{16} \mathrm{OH}^{-}$, and ${ }^{71} \mathrm{Ga}^{40} \mathrm{Ar}^{-}$(Hu et al., 2006).

In addition, the high variation of $\mathrm{Mg}$ in samples i.e., $\mathrm{B} 4$ and $\mathrm{C} 2$ may be attributed to the composed ions (i.e. ${ }^{25} \mathrm{Mg}^{35} \mathrm{Cl}^{-}$). In addition, the potential was hindered by the presence of molecular interferences $\left(\mathrm{C}_{2}^{+}, \mathrm{C}_{2} \mathrm{H}^{+}, \mathrm{C}_{2} \mathrm{H}^{2}, \mathrm{CN}^{+}\right.$and $\left.\mathrm{NaH}^{+}\right)$in the Ar-ICP system (Galy et al., 2001). A major error in $\mathrm{Cr}$ determination was due to the $\mathrm{Cr}$ loss incurred through volatilization during sample digestion. Many investigators have reported the volatility effect on $\mathrm{Cr}(\mathrm{VI})$ from the digestion and evaporation process. Interference problems due to argon gas, filter media, and/or acid-derived background ions, encountered in low-resolution ICP-MS, introduced additional errors in the $\mathrm{Cr}$ determination. Specifically, ${ }^{40} \mathrm{Ar}^{12} \mathrm{C}^{+},{ }^{38} \mathrm{Ar}^{14} \mathrm{~N}^{+},{ }^{35} \mathrm{Cl}^{16} \mathrm{OH}^{+}$, and ${ }^{35} \mathrm{Cl}^{17} \mathrm{O}^{+}$ions inhibit detection of the ${ }^{52} \mathrm{Cr}^{+}$ isotope. Even for relatively minor isotopes, there is interference (e.g., between ${ }^{53} \mathrm{Cr}^{+}$and ${ }^{37} \mathrm{Cl}^{16} \mathrm{O}^{+}$, and ${ }^{52} \mathrm{Cr}^{+}$and ${ }^{40} \mathrm{Arl}^{12} \mathrm{C}^{+}$) (Wang et al., 1999).

Table 3 lists the average relative analytic $\mathrm{M} / \mathrm{S}$ error of the different chemical species in all analyzed 


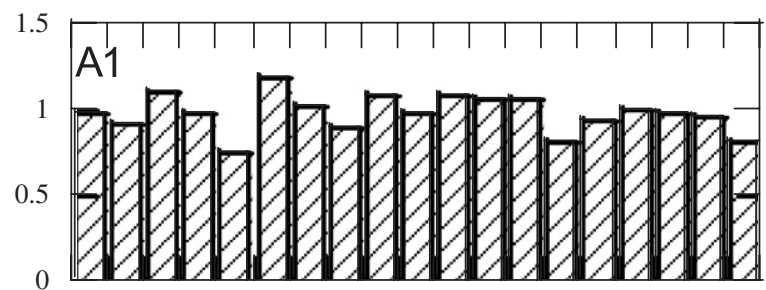

$\mathrm{Al} \mathrm{As} \mathrm{BaCaCd} \mathrm{CrCu} \mathrm{Fe} \mathrm{K} \mathrm{MgMnNa} \mathrm{Ni} \mathrm{Pb} \mathrm{Sb} \mathrm{Sr} \mathrm{Ti} \mathrm{V} \mathrm{Zn}$

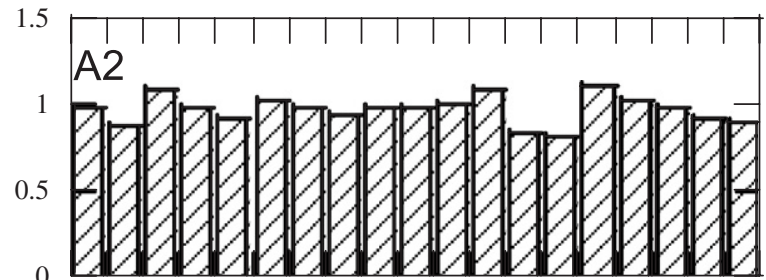

$\mathrm{Al} \mathrm{As} \mathrm{BaCaCd} \mathrm{Cr} \mathrm{Cu} F \mathrm{~K}$ MgMnNa Ni Pb Sb Sr Ti V Zn

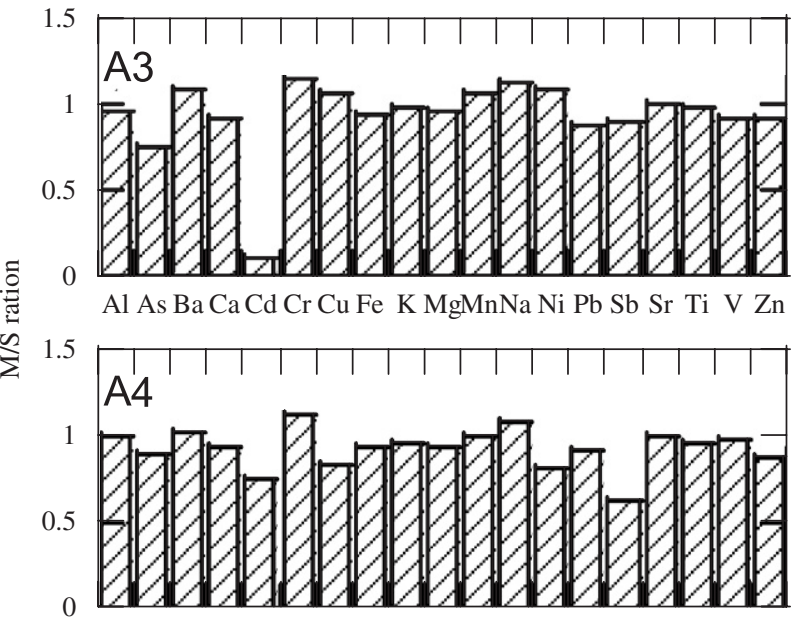

$\mathrm{Al} \mathrm{As} \mathrm{BaCaCd} \mathrm{Cr} \mathrm{Cu} F e \mathrm{~K}$ MgMnNa Ni Pb Sb Sr Ti V Zn
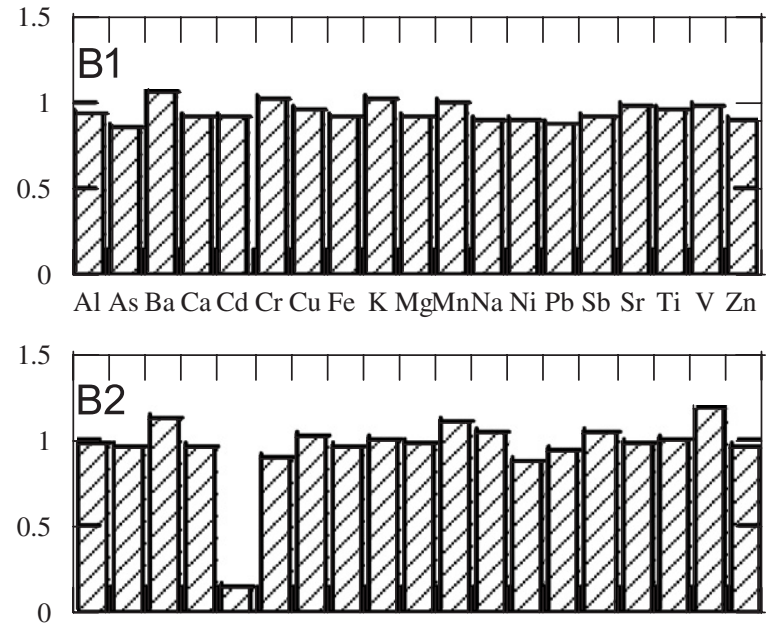

$\mathrm{Al} \mathrm{As} \mathrm{BaCaCd} \mathrm{Cr} \mathrm{Cu}$ Fe K MgMnNa Ni Pb Sb Sr Ti V Zn Elements

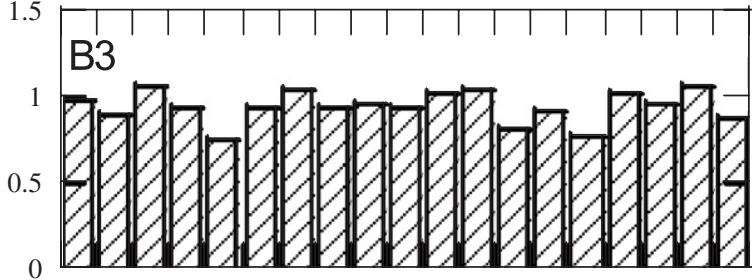

$\mathrm{Al} \mathrm{As} \mathrm{BaCaCd} \mathrm{Cr} \mathrm{Cu} \mathrm{Fe} \mathrm{K} \mathrm{MgMnNa} \mathrm{Ni} \mathrm{Pb} \mathrm{Sb} \mathrm{Sr} \mathrm{Ti} \mathrm{V}$ Zn

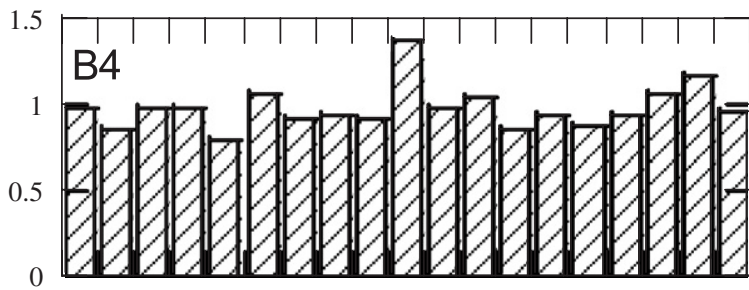

$\mathrm{Al} \mathrm{As} \mathrm{BaCaCd} \mathrm{Cr} \mathrm{Cu} F e \mathrm{~K} \mathrm{MgMnNa} \mathrm{Ni} \mathrm{Pb} \mathrm{Sb} \mathrm{Sr} \mathrm{Ti} V \mathrm{Zn}$
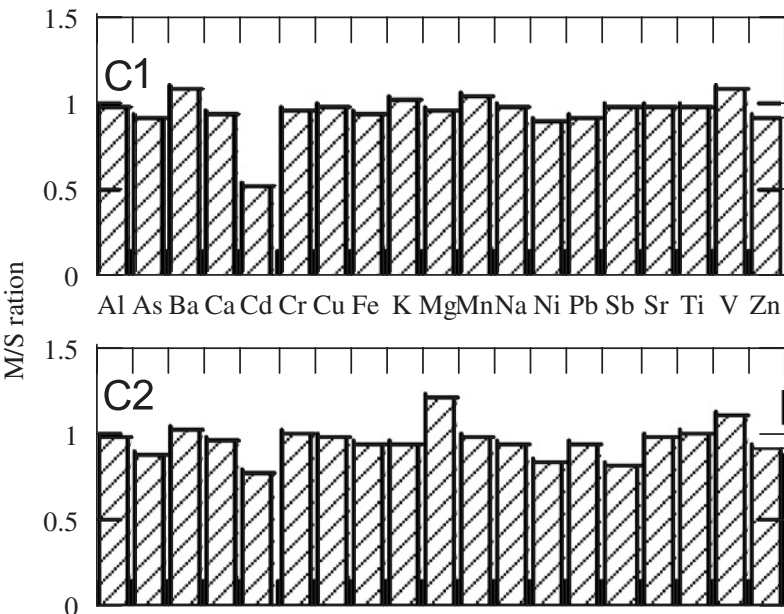

Al As Ba Ca Cd Cr Cu Fe K MgMnNa Ni Pb Sb Sr Ti V Zn
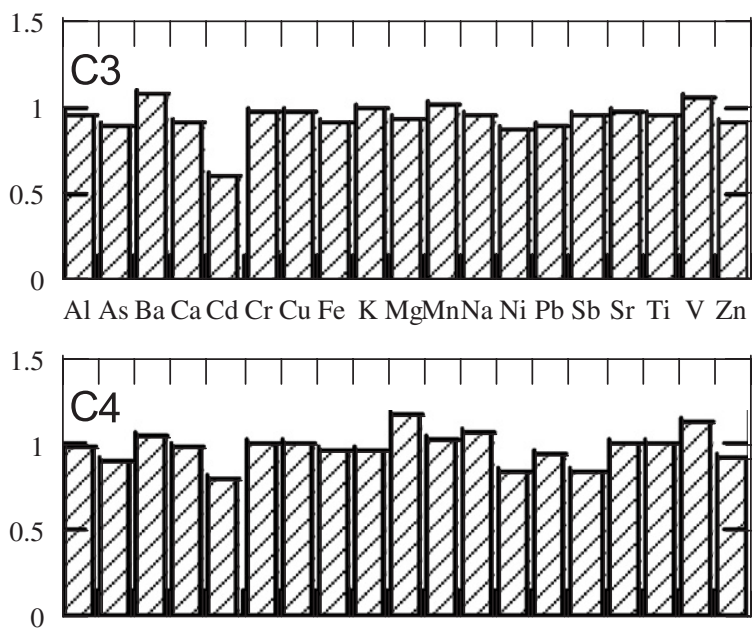

$\mathrm{Al} \mathrm{As} \mathrm{BaCaCd} \mathrm{Cr} \mathrm{Cu} F \mathrm{~K}$ KgMnNa Ni Pb Sb Sr Ti V Zn Elements

Fig. 1. $\mathrm{M} / \mathrm{S}$ (measured-to-stimulated) ratio of elemental compositions for 25 samples. 


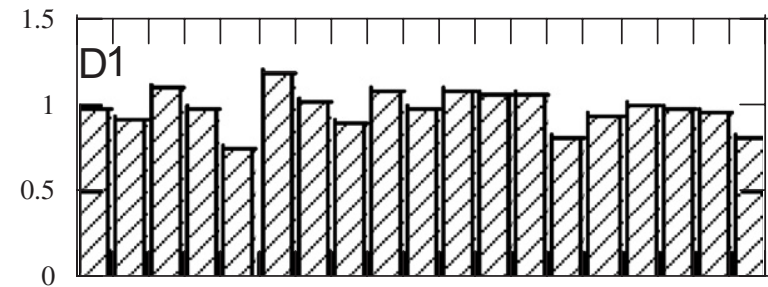

Al As Ba CaCd Cr Cu Fe K MgMnNa Ni Pb Sb Sr Ti V Zn

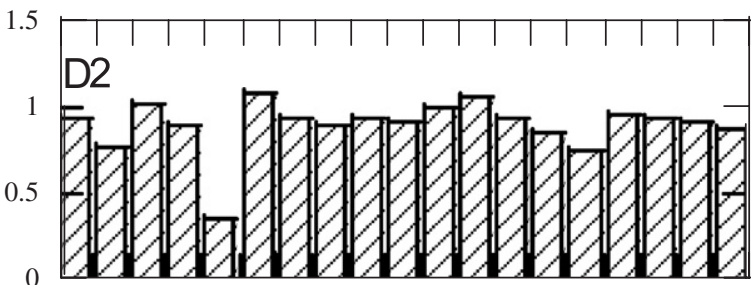

$\mathrm{Al} \mathrm{As} \mathrm{BaCaCd} \mathrm{Cr} \mathrm{Cu} F \mathrm{Ke} \mathrm{MgMnNa} \mathrm{Ni} \mathrm{Pb} \mathrm{Sb} \mathrm{Sr} \mathrm{Ti}$ V Zn

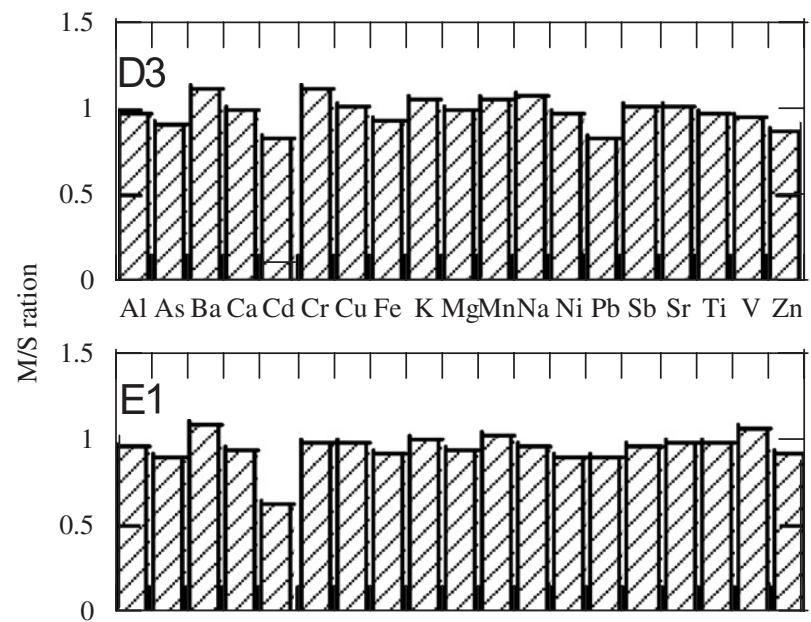

$\mathrm{Al} \mathrm{As} \mathrm{BaCaCd} \mathrm{CrCu} \mathrm{Fe} \mathrm{K} \mathrm{MgMnNa} \mathrm{Ni} \mathrm{Pb} \mathrm{Sb} \mathrm{Sr} \mathrm{Ti} \mathrm{V} \mathrm{Zn}$
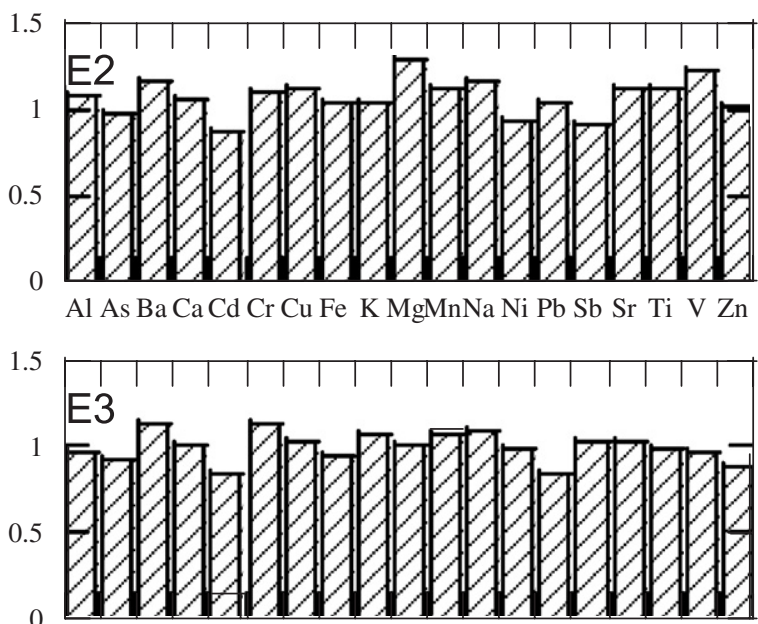

Al As Ba CaCd Cr Cu Fe K MgMnNa Ni Pb Sb Sr Ti V Zn Elements

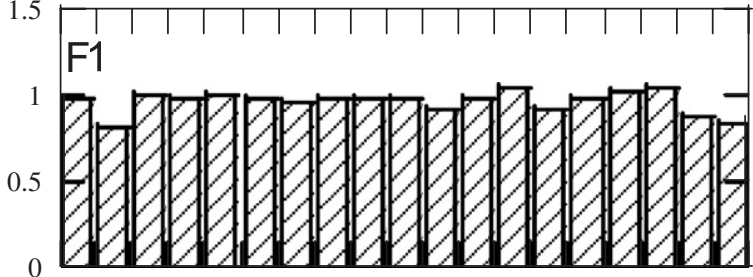

Al As Ba Ca Cd Cr Cu Fe K MgMnNa Ni Pb Sb Sr Ti V Zn

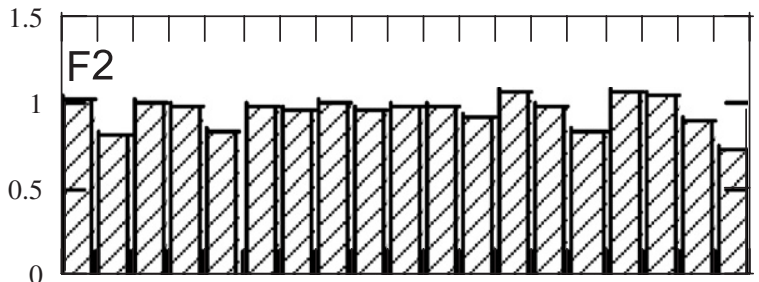

$\mathrm{Al} \mathrm{As} \mathrm{BaCaCd} \mathrm{Cr} \mathrm{Cu}$ Fe K MgMnNa Ni Pb Sb Sr Ti V Zn

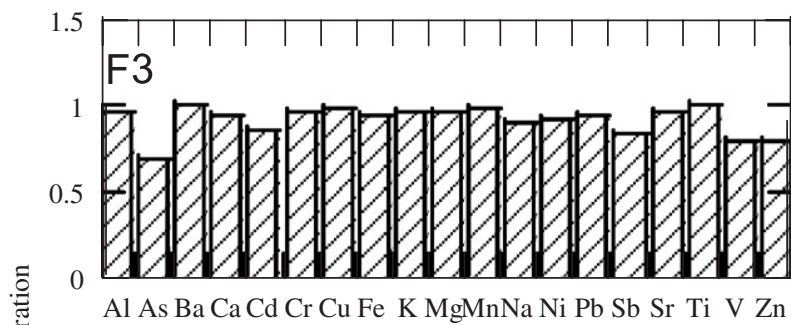

$\stackrel{2}{\sum} 1.5$

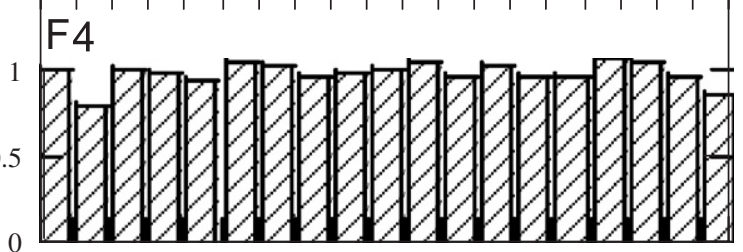

$\mathrm{Al} \mathrm{As} \mathrm{BaCaCd} \mathrm{Cr} \mathrm{Cu} F \mathrm{~K}$ KgMnNa Ni Pb Sb Sr Ti V Zn

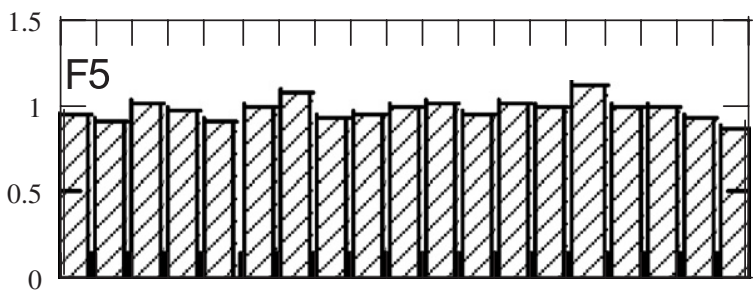

$\mathrm{Al} \mathrm{As} \mathrm{BaCaCd} \mathrm{Cr} \mathrm{Cu} F \mathrm{~K}$ MgMnNa Ni Pb Sb Sr Ti V Zn

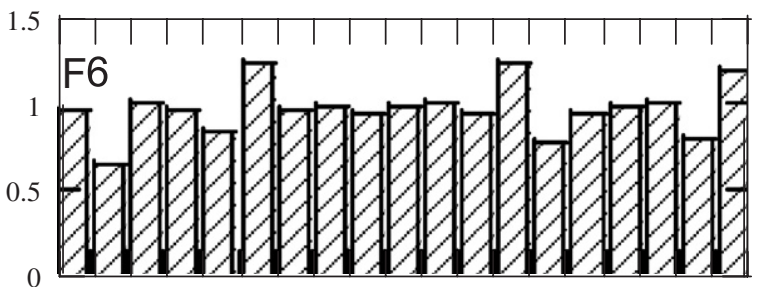

Al As Ba CaCd Cr Cu Fe K MgMnNa Ni Pb Sb Sr Ti V Zn Elements 
sample groups. As, $\mathrm{Cd}, \mathrm{Cr}, \mathrm{Ni}, \mathrm{Pb}, \mathrm{Sb}, \mathrm{V}$, and $\mathrm{Zn}$ analysis indicated high relative analysis error, especially $\mathrm{Cd}$. A total relative analysis error of the 19 element species was $1.46-1.79$ for the six group's particulate samples. There were insignificant differences between the six group samples in relative analysis error, but there were high errors in the A groups which may be attributed to three minor sources.

Lower measured recoveries in the Cd data (Fig. 1) may indicate the insensitivity of applied analytical techniques to trace amounts of chemical species. Based on Fig. 1 (E group), the sample mass did not significantly effect the analysis of sample composi-

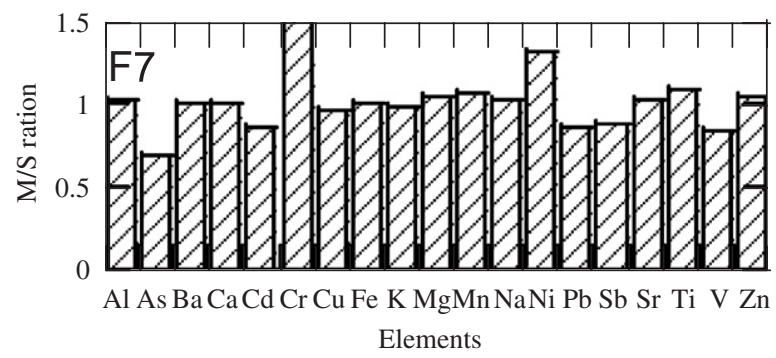

Fig. 1. (Continued) tions, yet some elements had high variation i.e., $\mathrm{Cd}$ in $\mathrm{E} 1$, and $\mathrm{Mg}$ and $\mathrm{V}$ in $\mathrm{E} 2$.

\subsection{Influence of analytical data's quality on $C M B$ receptor model analysis}

In a case study, the standard deviation for $\mathrm{Al}$ and $\mathrm{Fe}$ (non-marker species) was changed. The range of $5-30 \%$ corresponding to the change of source contribution was changed to $1-16 \%$ and all parameters (i.e., PMT, $r^{2}$ and $\chi^{2}$, etc.) fit the criteria of the model simulation. When the standard deviation of species analysis was over $30 \%$ the results of the model simulation were affected and did not meet the criteria. But few high standard deviations of nonmark species are measured in a high quality laboratory. Therefore, the non-mark species are not mentioned in the study (data not shown).

Table 4(a) shows the source contribution of each sample and the poor model identification for A3, B2, B4, C1, D2, E2 etc. Based on Fig. 1, the high analytic differences in sample composition response via the CMB model gave a low source contribution analytically. Most of samples had low Cd recovery in this study. In order to attempt to improve the source identification and fit the statistical criteria of

Table 3

Relative analytic error of elements for different sample groups

\begin{tabular}{|c|c|c|c|c|c|c|c|}
\hline Element & $\begin{array}{l}\text { A } \\
n=4\end{array}$ & $\begin{array}{l}\mathrm{B} \\
n=4\end{array}$ & $\begin{array}{l}\mathrm{C} \\
n=4\end{array}$ & $\begin{array}{l}\mathrm{D} \\
n=3\end{array}$ & $\begin{array}{l}\mathrm{E} \\
n=3\end{array}$ & $\begin{array}{l}\mathrm{F} \\
n=7\end{array}$ & Average \\
\hline $\mathrm{Al}$ & $0.02 \pm 0.02$ & $0.03 \pm 0.02$ & $0.03 \pm 0.01$ & $0.03 \pm 0.03$ & $0.06 \pm 0.03$ & $0.03 \pm 0.02$ & 0.03 \\
\hline As & $0.14 \pm 0.07$ & $0.11 \pm 0.04$ & $0.11 \pm 0.02$ & $0.13 \pm 0.08$ & $0.07 \pm 0.05$ & $0.24 \pm 0.09$ & 0.13 \\
\hline $\mathrm{Ba}$ & $0.08 \pm 0.04$ & $0.07 \pm 0.05$ & $0.06 \pm 0.03$ & $0.09 \pm 0.06$ & $0.12 \pm 0.04$ & $0.01 \pm 0.00$ & 0.07 \\
\hline $\mathrm{Ca}$ & $0.05 \pm 0.04$ & $0.05 \pm 0.03$ & $0.06 \pm 0.02$ & $0.04 \pm 0.06$ & $0.05 \pm 0.04$ & $0.03 \pm 0.02$ & 0.05 \\
\hline $\mathrm{Cd}$ & $0.37 \pm 0.36$ & $0.35 \pm 0.35$ & $0.32 \pm 0.13$ & $0.33 \pm 0.28$ & $0.22 \pm 0.14$ & $0.11 \pm 0.06$ & 0.28 \\
\hline $\mathrm{Cr}$ & $0.12 \pm 0.07$ & $0.07 \pm 0.03$ & $0.02 \pm 0.02$ & $0.12 \pm 0.03$ & $0.08 \pm 0.06$ & $0.13 \pm 0.19$ & 0.09 \\
\hline $\mathrm{Cu}$ & $0.07 \pm 0.07$ & $0.05 \pm 0.03$ & $0.02 \pm 0.01$ & $0.04 \pm 0.02$ & $0.06 \pm 0.06$ & $0.04 \pm 0.02$ & 0.05 \\
\hline $\mathrm{Fe}$ & $0.07 \pm 0.02$ & $0.07 \pm 0.02$ & $0.07 \pm 0.01$ & $0.08 \pm 0.02$ & $0.07 \pm 0.02$ & $0.04 \pm 0.03$ & 0.07 \\
\hline $\mathrm{K}$ & $0.05 \pm 0.03$ & $0.04 \pm 0.03$ & $0.04 \pm 0.02$ & $0.07 \pm 0.00$ & $0.04 \pm 0.03$ & $0.04 \pm 0.02$ & 0.05 \\
\hline $\mathrm{Mg}$ & $0.04 \pm 0.03$ & $0.14 \pm 0.17$ & $0.12 \pm 0.08$ & $0.03 \pm 0.05$ & $0.12 \pm 0.16$ & $0.02 \pm 0.02$ & 0.08 \\
\hline $\mathrm{Mn}$ & $0.04 \pm 0.04$ & $0.04 \pm 0.04$ & $0.03 \pm 0.02$ & $0.04 \pm 0.04$ & $0.07 \pm 0.05$ & $0.03 \pm 0.03$ & 0.04 \\
\hline $\mathrm{Na}$ & $0.09 \pm 0.03$ & $0.06 \pm 0.03$ & $0.05 \pm 0.02$ & $0.08 \pm 0.01$ & $0.10 \pm 0.06$ & $0.06 \pm 0.03$ & 0.07 \\
\hline $\mathrm{Ni}$ & $0.13 \pm 0.06$ & $0.14 \pm 0.04$ & $0.14 \pm 0.03$ & $0.03 \pm 0.03$ & $0.06 \pm 0.05$ & $0.11 \pm 0.12$ & 0.10 \\
\hline $\mathrm{Pb}$ & $0.14 \pm 0.05$ & $0.09 \pm 0.03$ & $0.08 \pm 0.02$ & $0.16 \pm 0.02$ & $0.11 \pm 0.07$ & $0.09 \pm 0.08$ & 0.11 \\
\hline $\mathrm{Sb}$ & $0.16 \pm 0.14$ & $0.12 \pm 0.08$ & $0.11 \pm 0.08$ & $0.09 \pm 0.013$ & $0.05 \pm 0.04$ & $0.10 \pm 0.06$ & 0.11 \\
\hline $\mathrm{Sr}$ & $0.01 \pm 0.01$ & $0.03 \pm 0.02$ & $0.01 \pm 0.01$ & $0.03 \pm 0.01$ & $0.06 \pm 0.06$ & $0.04 \pm 0.02$ & 0.03 \\
\hline $\mathrm{Ti}$ & $0.03 \pm 0.01$ & $0.05 \pm 0.03$ & $0.02 \pm 0.01$ & $0.03 \pm 0.04$ & $0.05 \pm 0.06$ & $0.04 \pm 0.03$ & 0.04 \\
\hline $\mathrm{V}$ & $0.06 \pm 0.03$ & $0.11 \pm 0.08$ & $0.09 \pm 0.03$ & $0.06 \pm 0.03$ & $0.11 \pm 0.10$ & $0.13 \pm 0.06$ & 0.09 \\
\hline $\mathrm{Zn}$ & $0.12 \pm 0.04$ & $0.08 \pm 0.04$ & $0.08 \pm 0.00$ & $0.13 \pm 0.00$ & $0.08 \pm 0.06$ & $0.17 \pm 0.07$ & 0.11 \\
\hline Sum & $1.79 \pm 0.50$ & $1.70 \pm 0.27$ & $1.46 \pm 0.06$ & $1.61 \pm 0.61$ & $1.58 \pm 0.41$ & $1.46 \pm 0.15$ & 1.60 \\
\hline
\end{tabular}

Analysis by ICP-AES/ICP-MS.

Relative analysis error $\sum\left|\frac{\mathrm{M}}{\mathrm{S}}-1\right| / n, n$ is number of samples. 
Table 4

\begin{tabular}{llllll}
\hline Composites & SRM 1648 & BCR 176 & SRM 2709 & SRM 1633b & $\begin{array}{l}\text { Analytic } \\
\text { percentage }\end{array}$
\end{tabular}

(a) CMB simulation by using the analysis results of ICP-MS

$\begin{array}{lc}\text { A1 } & 69.3 \pm 2.9(99)^{\mathrm{a}} \\ \text { A2 } & -\mathbf{3 . 1} \pm \mathbf{5 . 7}(\mathbf{- 3 1})^{\mathrm{b}} \\ \text { A3 } & \mathbf{2 0 . 6} \pm \mathbf{1 . 2}(\mathbf{2 0 6 )} \\ \text { A4 } & 11.4 \pm 1.6(114) \\ \text { B1 } & 35.1 \pm 3.3(88) \\ \text { B2 } & \mathbf{4 5 . 9} \pm \mathbf{2 . 3 ( 4 5 9 )} \\ \text { B3 } & 22.6 \pm 1.7(113) \\ \text { B4 } & \mathbf{2 2 . 3} \pm \mathbf{2 . 6}(\mathbf{7 4 )} \\ \text { C1 } & 38.4 \pm 3.2(116) \\ \text { C2 } & 1.4 \pm 2.9(-) \\ \text { C3 } & 31.4 \pm 3.3(95) \\ \text { C4 } & 31.7 \pm 2.7(96) \\ \text { D1 } & 35.5 \pm 3.9(89) \\ \text { D2 } & 45.7 \pm 2.2(114) \\ \text { D3 } & 37.8 \pm 3.8(95) \\ \text { E1 } & 24.0 \pm 8.0(96) \\ \text { E2 } & 25.1 \pm 2.2(101) \\ \text { E3 } & 23.6 \pm 3.4(95) \\ \text { F1 } & 34.8 \pm 3.0(91) \\ \text { F2 } & 35.4 \pm 2.4(104) \\ \text { F3 } & \mathbf{1 5 . 5} \pm \mathbf{3 . 4}(\mathbf{1 2 4}) \\ \text { F4 } & 17.7 \pm 3.0(95) \\ \text { F5 } & 29.3 \pm 3.0(110) \\ \text { F6 } & \mathbf{9 . 0} \pm \mathbf{2 . 2}(\mathbf{7 5 )} \\ \text { F7 } & 24.4 \pm 2.5(94) \\ & \end{array}$

$8.3 \pm 0.8(83)$
$73.6 \pm 2.7(105)$
$-\mathbf{0 . 5} \pm \mathbf{0 . 2}(-\mathbf{5})$
$\mathbf{7 . 7} \pm \mathbf{0 . 5}(\mathbf{7 7 )}$
$32.7 \pm 1.2(109)$
$\mathbf{1 . 8} \pm \mathbf{0 . 6}(\mathbf{4 . 6})$
$\mathbf{7 . 0} \pm \mathbf{0 . 5}(\mathbf{7 0 )}$
$16.1 \pm 1.1(81)$
$\mathbf{2 1 . 8} \pm \mathbf{1 . 2}(\mathbf{6 6 )}$
$26.5 \pm 1.2(80)$
$0.3 \pm 1.2(-)$
$\mathbf{2 6 . 2} \pm \mathbf{1 . 1}(\mathbf{8 0 )}$
$38.7 \pm 1.7(97)$
$\mathbf{0 . 8} \pm \mathbf{0 . 5}(\mathbf{8 )}$
$9.6 \pm 1.4(96)$
$22.2 \pm 3.1(89)$
$\mathbf{1 9 . 4} \pm \mathbf{0 . 9}(\mathbf{7 7})$
$23.8 \pm 1.4(95)$
$\mathbf{1 7 . 7} \pm \mathbf{1 . 2}(\mathbf{1 3 2})$
$25.6 \pm 0.9(98)$
$43.3 \pm 1.5(106)$
$36.9 \pm 1.2(111)$
$28.8 \pm 1.2(114)$
$13.1 \pm 0.8(104)$
$13.5 \pm 0.9(98)$

9

$\mathbf{1 3 . 4} \pm \mathbf{2 . 6}(\mathbf{1 3 4})$
$\mathbf{1 4 . 9} \pm \mathbf{6 . 1}(\mathbf{1 4 9 )}$
$78.4 \pm 2.3(111)$
$11.9 \pm 1.9(119)$
$21.4 \pm 3.4(107)$
$\mathbf{5 0 . 8} \pm \mathbf{3 . 3}(\mathbf{1 6 9})$
$42.1 \pm 3.1(105)$
$\mathbf{3 5 . 2} \pm \mathbf{2 . 6}(\mathbf{3 5 2})$
$\mathbf{4 4 . 3} \pm \mathbf{3 . 9}(\mathbf{1 3 4})$
$\mathbf{4 6 . 5} \pm \mathbf{3 . 3}(\mathbf{1 4 1})$
$36.1 \pm 3.8(109)$
$\mathbf{1 5 . 1} \pm \mathbf{3 . 4}(-)$
$\mathbf{1 6 . 9} \pm \mathbf{4 . 0}(\mathbf{1 6 9})$
$47.5 \pm 2.7(119)$
$\mathbf{1 3 . 6} \pm \mathbf{3 . 5}(\mathbf{1 3 6})$
$\mathbf{3 1 . 8} \pm \mathbf{9 . 3}(\mathbf{1 2 7})$
$\mathbf{3 5 . 8} \pm \mathbf{3 . 2}(\mathbf{1 4 3 )}$
$29.6 \pm 3.5(118)$
$\mathbf{1 1 . 5} \pm \mathbf{3 . 1}(\mathbf{6 5 )}$
$\mathbf{1 2 . 5} \pm \mathbf{2 . 7}(\mathbf{6 8 )}$
$\mathbf{2 0 . 2} \pm \mathbf{4 . 0}(\mathbf{7 3})$
$\mathbf{1 6 . 9} \pm \mathbf{3 . 4}(\mathbf{7 3})$
$\mathbf{2 . 1} \pm \mathbf{3 . 4}(\mathbf{2 0})$
$39.3 \pm 2.7(105)$
$36.6 \pm 2.9(106)$

16

\begin{tabular}{rrr}
$8.9 \pm 1.2(89)$ & 100.4 & 2.10 \\
$11.5 \pm 3.2(115)$ & 93.2 & 1.15 \\
$\mathbf{1 . 5} \pm \mathbf{1 . 0}(\mathbf{1 5 )}$ & $\mathbf{1 2 0 . 8}$ & $\mathbf{2 5 . 2 5}$ \\
$69.0 \pm 2.1(99)$ & 99.5 & 1.71 \\
$10.8 \pm 1.4(108)$ & 92.4 & 1.33 \\
$\mathbf{1 . 5} \pm \mathbf{1 . 6}(\mathbf{7})$ & $\mathbf{1 4 1 . 3}$ & $\mathbf{2 8 . 0 5}$ \\
$28.3 \pm 1.8(94)$ & 101.7 & 1.11 \\
$\mathbf{2 6 . 4} \pm \mathbf{1 . 5}(\mathbf{6 6 )}$ & 111.6 & $\mathbf{4 . 6 3}$ \\
$-4.5 \pm 1.8(-)$ & 109.0 & $\mathbf{4 . 9 5}$ \\
$\mathbf{2 5 . 5} \pm \mathbf{1 . 8}(\mathbf{7 7 )}$ & 108.9 & 1.73 \\
$32.1 \pm 1.9(97)$ & 96.8 & 0.84 \\
$27.0 \pm 1.9(82)$ & 109.8 & 1.34 \\
$8.9 \pm 1.9(89)$ & 101.2 & 1.94 \\
$\mathbf{6 . 0} \pm \mathbf{1 . 4}(\mathbf{6 0 )}$ & 105.6 & $\mathbf{7 . 9 2}$ \\
$38.9 \pm 2.2(97)$ & 99.9 & 0.73 \\
$22.0 \pm 4.1(88)$ & 99.7 & 0.42 \\
$\mathbf{1 9 . 8} \pm \mathbf{1 . 7}(\mathbf{7 9 )}$ & 120.0 & 1.19 \\
$23.0 \pm 1.8(92)$ & 100.6 & 1.61 \\
$35.9 \pm 1.8(118)$ & 88.3 & 3.84 \\
$\mathbf{2 6 . 5} \pm \mathbf{1 . 5}(\mathbf{1 2 4})$ & 93.7 & 3.90 \\
$21.1 \pm 1.9(110)$ & 88.5 & 3.20 \\
$28.5 \pm 1.8(115)$ & 91.5 & 3.58 \\
$39.8 \pm 1.9(105)$ & 88.6 & $\mathbf{4 . 0 6}$ \\
$38.3 \pm 1.6(102)$ & 94.3 & $\mathbf{4 . 7 7}$ \\
$25.5 \pm 1.6(99)$ & 99.2 & $\mathbf{6 . 2 1}$ \\
7 & 2 & 8 \\
\hline & &
\end{tabular}

$8.9+1.2(89) \quad 100.3$

$9.6 \pm 2.9(96) \quad 96.2$

$2.1 \pm 1.0(21)$

$69.3 \pm 2.1(99)$

$10.4 \pm 1.4(104)$

$3.7 \pm 1.5(19)$

$28.2 \pm 1.8(94)$

$26.2 \pm 1.5$ (66)

$-4.3 \pm 1.8(-)$

$25.6 \pm 1.8(78)$

$32.0 \pm 1.8(97)$

$27.0 \pm 1.9(82)$

$8.9 \pm 1.8(90)$

$6.4 \pm 1.4$ (64)

$38.3 \pm 2.1(96)$

$21.6 \pm 3.8(87)$

$20.0 \pm 1.8(80)$

$23.0 \pm 1.8(92)$

$34.6 \pm 1.7$ (113)

$26.2 \pm 1.5$ (122)

$20.9 \pm 1.8(109)$

$28.1 \pm 1.8(113)$

$39.5 \pm 1.8(104)$

$37.6 \pm 1.6(100)$

$25.2 \pm 1.5$ (97)
96.2
$\mathbf{1 2 0 .}$

99.1

93.2

137.1

102.0

112.0

108.7

108.6

97.2

109.0

101.5

104.6

100.8

100.5

119.1

100.8

89.6

94.0

88.8

92.1

89.0

94.8

99.6
2.10

25.25

1.71

28.05

1.11

4.95

1.73

0.84

1.94

7.92

0.42

1.19

1.61

3.90

3.20

3.58

4.06

4.77
6.21

8

1.77

1.20

27.02

1.47

1.31

30.50

1.08

3.90

4.19

1.45

0.71

1.23

1.71

8.04

0.68

0.36

1.16

1.39

3.38

3.32

2.71

3.04

3.44

4.35

5.71

6

Out of criteria (\#) 5 
Table 4 (continued)

\begin{tabular}{|c|c|c|c|c|c|c|}
\hline Composites & SRM 1648 & BCR 176 & SRM 2709 & SRM 1633b & $\begin{array}{l}\text { Analytic } \\
\text { percentage }\end{array}$ & $\chi^{2}$ \\
\hline \multicolumn{7}{|c|}{ (c) CMB simulation by using the analysis results of ICP-MS, IC and spectrophotometer } \\
\hline A1 & $70.9 \pm 2.8(101)^{\mathrm{a}}$ & $8.8 \pm 0.8(88)$ & $10.6 \pm 2.1(106)$ & $9.7 \pm 1.2(97)$ & 98.5 & 1.85 \\
\hline A2 & $1.6 \pm 4.8(16)$ & $73.2 \pm 2.3(104)$ & $10.5 \pm 3.3(105)$ & $11.1 \pm 2.7(111)$ & 92.6 & 1.20 \\
\hline A3 & $18.3 \pm 1.0(183)^{\mathrm{b}}$ & $-0.1 \pm 0.2(-1)$ & $78.8 \pm 2.1(113)$ & $3.0 \pm 1.0(30)$ & 117.0 & 26.95 \\
\hline A4 & $10.9 \pm 1.3(109)$ & $8.0 \pm 0.4(80)$ & $11.6 \pm 1.8(116)$ & $69.4 \pm 2.1$ (99) & 98.3 & 1.49 \\
\hline $\mathrm{B} 1$ & $36.6 \pm 3.0(92)$ & $32.3 \pm 1.1(108)$ & $20.5 \pm 2.5(102)$ & $10.7 \pm 1.3$ & 92.7 & 1.25 \\
\hline $\mathrm{B} 2$ & $44.9 \pm 2.1(449)$ & $4.5 \pm 0.6(11.3)$ & $42.5 \pm 2.6(141)$ & $8.1 \pm 1.6(41)$ & 123.1 & 31.71 \\
\hline B3 & $22.9 \pm 7.5(114)$ & $7.3 \pm 0.5(73)$ & $40.6 \pm 2.7(102)$ & $29.2 \pm 1.8(98)$ & 99.9 & 1.22 \\
\hline B4 & $24.0 \pm 2.4(80)$ & $18.5 \pm 1.1(92)$ & $29.4 \pm 2.2(294)$ & $28.1 \pm 1.6(70)$ & 105.7 & 6.37 \\
\hline $\mathrm{C} 1$ & $40.3 \pm 2.9(122)$ & $24.6 \pm 1.2(75)$ & $37.3 \pm 2.9(113)$ & $-2.3 \pm 1.7$ & 102.4 & 4.53 \\
\hline $\mathrm{C} 2$ & $1.2 \pm 2.4$ & $29.0 \pm 1.1(88)$ & $42.1 \pm 2.8(127)$ & $27.8 \pm 1.8(84)$ & 103.6 & 2.22 \\
\hline $\mathrm{C} 3$ & $32.2 \pm 1.9(98)$ & $0.0 \pm 0.4$ & $34.9 \pm 2.8(106)$ & $32.9 \pm 1.7(100)$ & 95.9 & 0.75 \\
\hline $\mathrm{C} 4$ & $34.9 \pm 2.9(106)$ & $29.2 \pm 1.2(89)$ & $5.2 \pm 2.7$ & $30.7 \pm 2.0(93)$ & 101.4 & 2.12 \\
\hline D1 & $36.5 \pm 3.6(91)$ & $41.7 \pm 1.6(104)$ & $10.6 \pm 2.6(106)$ & $11.2 \pm 1.8(112)$ & 96.4 & 1.87 \\
\hline D2 & $45.3 \pm 1.9(113)$ & $1.6 \pm 0.4(16)$ & $45.6 \pm 2.4(114)$ & $7.5 \pm 1.4(75)$ & 101.9 & 8.04 \\
\hline D3 & $40.1 \pm 2.8(100)$ & $9.8 \pm 1.0(98)$ & $10.3 \pm 2.7(103)$ & $39.8 \pm 2.1$ (99) & 98.3 & 0.81 \\
\hline E1 & $25.5 \pm 4.2(102)$ & $24.1 \pm 2.3(96)$ & $24.9 \pm 4.2(100)$ & $25.6 \pm 3.6(102)$ & 94.8 & 0.43 \\
\hline E2 & $25.5 \pm 4.2(102)$ & $24.1 \pm 2.3(96)$ & $24.9 \pm 4.2(100)$ & $25.6 \pm 3.6(102)$ & 109.6 & 2.9 \\
\hline E3 & $22.5 \pm 2.6(90)$ & $21.9 \pm 1.1(88)$ & $34.6 \pm 2.8(138)$ & $21.0 \pm 1.7(84)$ & 104.7 & 1.80 \\
\hline $\mathrm{F} 1$ & $37.1 \pm 2.2(97)$ & $16.3 \pm 1.0(121)$ & $12.7 \pm 2.4(72)$ & $33.9 \pm 1.6(111)$ & 90.5 & 3.26 \\
\hline $\mathrm{F} 2$ & $35.9 \pm 2.0(105)$ & $25.2 \pm 0.8$ & $12.9 \pm 2.2(71)$ & $26.0 \pm 1.5(121)$ & 94.5 & 3.16 \\
\hline F3 & $15.2 \pm 2.6(122)$ & $41.8 \pm 1.3(102)$ & $23.4 \pm 2.9(85)$ & $19.6 \pm 1.7(102)$ & 90.6 & 2.68 \\
\hline $\mathrm{F} 4$ & $18.7 \pm 2.3(100)$ & $36.0 \pm 1.1(108)$ & $17.6 \pm 2.6(76)$ & $27.7 \pm 1.7(112)$ & 92.7 & 2.90 \\
\hline F5 & $29.0 \pm 2.3(109)$ & $27.6 \pm 1.0(110)$ & $5.1 \pm 2.6(49)$ & $38.3 \pm 1.8(101)$ & 90.8 & 3.40 \\
\hline F6 & $11.4 \pm 1.3(95)$ & $12.9 \pm 0.6(101)$ & $37.9 \pm 2.4(101)$ & $37.9 \pm 1.6(101)$ & 94.4 & 4.12 \\
\hline F7 & $25.9 \pm 1.8(100)$ & $13.9 \pm 0.8(101)$ & $33.8 \pm 2.4(98)$ & $26.4 \pm 1.5(102)$ & 97.6 & 5.52 \\
\hline Out of criteria (\#) & 4 & 6 & 9 & 6 & 1 & 7 \\
\hline
\end{tabular}

\footnotetext{
${ }^{a}$ Value in the parenthesis implies the relative percentage of model: simulation(mass of model simulation/mass in the particulate sample) $\times 100 \%$.

${ }^{\mathrm{b}}$ Bold mark indicates the data could not fit the criteria of model.
}

the $\mathrm{CMB}$ model, $\mathrm{Cl}^{-}, \mathrm{F}^{-}$and $\mathrm{SO}_{4}^{2-}$ were analyzed by IC and combined with the other 19 elements analyzed by ICP-MS. The results of the CMB model are shown in Table 4(b). These three additional ionic species did not enhance the model in identifying the pollution source. When a total of 23 species including $\mathrm{Si}$ was analyzed and measured by $\mathrm{CMB}$, the model identification was enhanced significantly as shown in Table 4(c). Some of the samples (i.e., A3, B2, and C1), however, did not meet the model criteria. Therefore, the precision and accuracy of chemical sample species are important in the CMB model.

In order to improve the CMB source identification, some of the high variation and low recovery elements were removed from the chemical species i.e., Cd. Table 5 shows a typical case (A3); results indicated that the removal enhanced the identification of the CMB model significantly. Omitting the high variation and low recovery elements in most cases better enhanced the model identification than by adding more analyzed species in the samples. Therefore, the precision and accuracy of analyzed elements are one of important factors which affect the model analysis.

To understand the influence of measured analytical data on the CMB results, the chemical species in the source profiles were further classified according to their concentrations and relative significance.

One of the classic problems of CMB models is that for many species, the many source composition patterns are so similar that it is difficult to resolve the concentrations. As illustrated in Tables 3 and 4, species having higher relative concentrations in minor sources are not helpful because their concentrations in simulated samples may be dominated by other sources. In fact, good source profile precision and accuracy of unique species ("marker" species, $M / S \approx 1.0$ ) have a great influence on the resolving ability of the CMB model. 
Table 5

Sample A3 Cd removal from the element species

\begin{tabular}{|c|c|c|c|c|c|c|}
\hline Analytic instrument & SRM 1648 & BCR 176 & SRM 2709 & SRM 1633b & $\begin{array}{l}\text { Analytic } \\
\text { percentage }\end{array}$ & $\chi^{2}$ \\
\hline ICP-MS & $20.6 \pm 1.2(206)^{\mathrm{a}}$ & $-0.5 \pm 0.2(-5)$ & $78.4 \pm 2.3(112)$ & $1.5 \pm 1.0(15)$ & 120.8 & 25.25 \\
\hline ICP-MS (no Cd) & $6.3 \pm 1.7(63)$ & $10.7 \pm 0.7(107)$ & $75.3 \pm 2.7(108)$ & $7.7 \pm 1.2(77)$ & 100.6 & 3.1 \\
\hline $\mathrm{ICP}-\mathrm{MS}+\mathrm{IC}$ & $17.7 \pm 1.0(177)$ & $-0.1 \pm 0.2(-1)$ & $80.4 \pm 2.3(115)$ & $2.1 \pm 1.0(21)$ & 120.1 & 27.02 \\
\hline ICP-MS + IC (no Cd) & $7.4 \pm 1.3(74)$ & $10.4 \pm 0.6(104)$ & $74.3 \pm 2.4(106)$ & $7.9 \pm 1.1(79)$ & 100 & 2.86 \\
\hline $\mathrm{ICP}-\mathrm{MS}+\mathrm{IC}+\mathrm{Si}$ & $18.3 \pm 1.0(183)$ & $-0.1 \pm 0.2(-1)$ & $78.8 \pm 2.1(113)$ & $3.0 \pm 1.0(30)$ & 117.0 & 26.95 \\
\hline $\mathrm{ICP}-\mathrm{MS}+\mathrm{IC}+\mathrm{Si}($ no $\mathrm{Cd})$ & $7.5 \pm 1.3(75)$ & $10.0 \pm 0.6(100)$ & $75.2 \pm 2.6(107)$ & $7.3 \pm 1.1(73)$ & 101.6 & 2.76 \\
\hline
\end{tabular}

\footnotetext{
${ }^{\text {a }}$ Value in the parenthesis implies the relative percentage of model: simulation(mass of model simulation/mass in the particulate sample) $\times 100 \%$.
}

To evaluate the resolving ability of the CMB model on major, minor and non-existing sources, different groups' simulated samples were analyzed to apportion their source contributions, respectively. As seen in Table 4, the apportioned source contributions are generally in good agreement with the simulated values. On the other hand, significant fluctuations appeared on the estimated source apportions of minor sources, which may indicate the existence of a serious collinear problem. It was noticed that NIST SRM 1648 and BCR No. 176, or NIST SRM 1633b and NIST SRM 2709 were not adequately resolved from each other during the analysis, even though their compositions were not identical. In fact, the sum of these two group's estimated major source contributions very closely approximates that of simulated contributions in D1 sample.

A major challenge to the application of the CMB model is to distinguish the non-existing and unknown sources, which have been included in the source profile of the model (Christensen, 2004; Christensen and Gunst, 2004). It was found from Table 4, that the sources not actually present generally yielded contributions less than their standard errors except for the $\mathrm{C} 4$ sample. In $\mathrm{C} 4$, the source contribution of the soil standard was overestimated since its prominent species was in common with the coal fly ash standard. Actually, the profiles of all standard sources were significantly distinct from those of other sources. As a result, overestimating the number of sources had little effect on the calculated source contributions if the "marker" species of non-existing sources were included in the calculation of source contributions.

Generally, the problem of colinearity is caused by the intercorrelation among the called "independent species" (compositions of particulate samples). Although the intercorrelation among these variables is not extremely high, they usually significantly affect the results of CMB model simulation and lead to distortions in the results of the model. Therefore, some particulate source could be overestimation and some sources could be extinction while the colinearity problem in the pollution sources of particle. Table 6 shows the combined collinear source profiles which improved the model stimulation for $\mathrm{C} 1$ sample.

A quantitative deviation between the simulated and estimated source contributions can be calculated in terms of RPD (relative percentage difference):

$\operatorname{RPD}(\%)=\left\{\left|X_{S}-X_{E}\right|\right\} /\left\{\left(X_{S}+X_{E}\right) / 2\right\}$,

where $X_{\mathrm{S}}$ and $X_{\mathrm{E}}$ are the simulated and estimated source contributions, respectively.

It was found that the apportioned source's RPD values generally decrease with the extent of contributions. The RPD values of major sources can generally be controlled within $20 \%$. Large deviations can only be observed for minor sources and be attributed to inaccurate analytical data as well as colinearity among sources.

\subsection{Improving the CMB result by a two-step procedure}

Ideally, one should use concentrations of all measured species in receptor modeling to obtain the source strength; however, in testing the $\mathrm{CMB}$ method, we can use the $\mathrm{S} / \mathrm{M}$ concentration ratios of different species as a measure of the quality of the fit. A better $\mathrm{CMB}$ receptor model result can then be achieved by removing the data of species whose $\mathrm{M} / \mathrm{S}$ 
Table 6

A case study of combined colinearity sources

\begin{tabular}{|c|c|c|c|c|c|c|}
\hline Composites & SRM 1648 & BCR 176 & SRM 2709 & SRM 1633b & $\begin{array}{l}\text { Analytic } \\
\text { percentage }\end{array}$ & $\chi^{2}$ \\
\hline ICP-MS & $38.4 \pm 3.2(116)^{\mathrm{a}}$ & $21.8 \pm 1.2(66)$ & $44.3 \pm 3.9(134)$ & $-4.5 \pm 1.8(-)$ & 109.0 & 4.95 \\
\hline $\mathrm{ICP}-\mathrm{MS}+\mathrm{IC}$ & $37.4 \pm 2.8(113)$ & $22.3 \pm 1.2(68)$ & $44.6 \pm 3.8(135)$ & $-4.3 \pm 1.8(-)$ & 108.7 & 4.19 \\
\hline $\mathrm{ICP}-\mathrm{MS}+\mathrm{IC}+\mathrm{Si}$ & $40.3 \pm 2.9(122)$ & $24.6 \pm 1.2(75)$ & $37.3 \pm 2.9(113)$ & $-2.3 \pm 1.7$ & 102.4 & 4.53 \\
\hline & \multicolumn{2}{|c|}{ M1 } & \multicolumn{2}{|c|}{ M2 } & & \\
\hline ICP-MS & \multicolumn{2}{|c|}{$69.8 \pm 1.7(106)$} & \multicolumn{2}{|c|}{$30.2 \pm 0.6(92)$} & 101.3 & 3.63 \\
\hline $\mathrm{ICP}-\mathrm{MS}+\mathrm{IC}$ & \multirow{2}{*}{\multicolumn{2}{|c|}{$69.8 \pm 1.7(106)$}} & \multicolumn{2}{|c|}{$30.2 \pm 0.6(92)$} & 101.3 & 3.09 \\
\hline $\mathrm{ICP}-\mathrm{MS}+\mathrm{IC}+\mathrm{Si}$ & & & \multicolumn{2}{|c|}{$30.6 \pm 0.6(93)$} & 100.4 & 3.11 \\
\hline
\end{tabular}

M1: SRM 1648 and BCR 176; M2: SRM 2709 and SRM 1633b.

Bold mark indicates the data could not fit the criteria of model.

${ }^{a}$ Value in the parenthesis implies the relative percentage of model: simulation(mass of model simulation/mass in the particulate sample) $\times 100 \%$.

Table 7

Variations of $\chi^{2}$ values of prepared samples

\begin{tabular}{|c|c|c|c|c|}
\hline Sample no. & $\begin{array}{l}\text { All analyzed } \\
\text { chemical species }^{\text {a }}\end{array}$ & $|\mathrm{M} / \mathrm{S}-1| \leqslant \pm 20 \%$ & $|\mathrm{M} / \mathrm{S}-1| \leqslant \pm 15 \%$ & $|\mathrm{M} / \mathrm{S}-1| \leqslant \pm 10 \%$ \\
\hline Al & 1.85 & $1.22(22)^{\mathrm{b}}$ & 0.98 (19) & $0.86(16)$ \\
\hline $\mathrm{A} 2$ & 1.20 & $1.20(23)$ & $1.12(22)$ & 0.67 (19) \\
\hline A3 & 27.0 & $1.34(18)$ & $0.80(11)$ & $0.17(10)$ \\
\hline A4 & 1.49 & $1.41(21)$ & $1.24(19)$ & $1.24(19)$ \\
\hline $\mathrm{B} 1$ & 1.25 & $1.25(23)$ & $1.18(22)$ & $1.21(21)$ \\
\hline $\mathrm{B} 2$ & 31.7 & $2.82(16)$ & $0.41(6)$ & 0.78 (4) \\
\hline B3 & 1.22 & $1.21(22)$ & $1.15(21)$ & 0.87 (18) \\
\hline B4 & 6.37 & $3.78(22)$ & $0.58(21)$ & 0.15 (18) \\
\hline $\mathrm{Cl}$ & 4.53 & $1.06(21)$ & $1.13(20)$ & $0.93(17)$ \\
\hline $\mathrm{C} 2$ & 2.22 & $2.17(22)$ & $1.13(20)$ & $0.31(13)$ \\
\hline $\mathrm{C} 3$ & 0.75 & $0.73(22)$ & $0.73(22)$ & $0.68(21)$ \\
\hline $\mathrm{C} 4$ & 2.22 & $2.22(23)$ & $1.35(20)$ & $0.50(13)$ \\
\hline D1 & 1.87 & $1.65(22)$ & 0.52 (19) & 0.17 (16) \\
\hline D2 & 8.04 & $0.70(15)$ & $0.70(15)$ & $0.67(11)$ \\
\hline D3 & 0.81 & $0.81(23)$ & $0.42(20)$ & $0.41(16)$ \\
\hline E1 & 0.43 & $0.25(22)$ & $0.25(22)$ & $0.20(21)$ \\
\hline E2 & 2.90 & $2.90(23)$ & $0.63(17)$ & $0.45(14)$ \\
\hline E3 & 1.80 & $1.80(23)$ & $1.65(21)$ & 0.58 (18) \\
\hline $\mathrm{F} 1$ & 3.26 & $2.76(22)$ & $2.53(20)$ & $1.41(14)$ \\
\hline $\mathrm{F} 2$ & 3.16 & $3.16(23)$ & $2.74(21)$ & $1.86(19)$ \\
\hline F3 & 2.68 & $2.50(22)$ & $1.63(21)$ & $1.25(19)$ \\
\hline F4 & 2.90 & 2.69 (22) & $1.60(20)$ & $0.58(18)$ \\
\hline F5 & 3.40 & $3.30(22)$ & $2.83(20)$ & 0.98 (17) \\
\hline F6 & 4.72 & $2.12(21)$ & 1.99 (19) & $1.34(17)$ \\
\hline F7 & 7.52 & 1.78 (19) & 1.78 (19) & 0.74 (18) \\
\hline
\end{tabular}

a 23 analyzed chemical species of samples.

${ }^{\mathrm{b}}$ Data presented in parenthesis is the number of chemical species input for CMB model.

values are apparently deviating from 1. For example, in this experiment, $\mathrm{Cd}$ was the element whose analytical data was not accurate and should not be placed in the model analysis. Table 5 shows that when the low $\mathrm{Cd}$ recovery was not included in the A3 CMB model, there was a significant increase in the resolution of source contribution.

Table 7 shows the $\chi^{2}$ values of the primary CMB results analyzed from various samples and the removal of the chemical species from the input data 
with $(\mathrm{M} / \mathrm{S}-1)$ values over $\pm 20 \%, \pm 15 \%$ or $\pm 10 \%$, respectively. We demonstrate, through the examination of these samples, that the $\chi^{2}$ values decrease generally with removing the less precise chemical species from the model input data. Significant improvements were observed for samples of A3, B2, D2 and F7 (Fig. 1), in which data of the most eight inaccurate species $(|\mathrm{M} / \mathrm{S}-1| \leqslant 0.20)$ had been initially removed. However, insignificant improvements for $\chi^{2}$ values were also observed if further chemical species were removed $(|\mathrm{M} / \mathrm{S}-1| \leqslant$ 0.15). Obviously, the resolving ability of the model is affected by the precision and accuracy of the input data and can be improved by removing inaccurate analytical data.

We found that the reduction of inaccurate or lower precision analytical data not only improves the statistical deviation of the model result, but also increases the capability of individual source apportionment. However, further reducing the number of chemical species without any criteria may insignificantly improve the statistical fluctuation but at the same time degrade the resolution of source contributions. In this investigation, the optimal criteria to select the acceptable analytical data will be those important "marker" species of pollution sources with $\mathrm{M} / \mathrm{S}$ values close to one. The $\chi^{2}$ values of all $\mathrm{CMB}$ results should also be controlled within 4 . In real sample analysis, the actual source contributions are unknown; however, we still can predict the concentration of each species from the primary CMB results and estimate the predicted-to-measured ratio as data acceptable criteria.

As a result, a two-step modeling procedure, which first compares the measured analytical data to the one that was calculated from source contributions of primary CMB results, and then eliminates those non-marker and lower precise chemical species improved the capability of CMB model analysis. While the proposed two-step CMB modeling approach cannot be directly verified in real sample analysis, the evaluation of randomly prepared samples in group "F" may give some insight into the effectiveness of this procedure.

\section{Conclusions}

A set of simple four-source simulated samples was systematically employed to test the capability of CMB receptor model. These tests with 23 commonly measured chemical species showed that different source-types can be apportioned with uncertainties less than $20 \%$ if accurate determinations of chemical species in both source and ambient samples are performed. Furthermore, better performance was demonstrated with the presence of unique "marker" species such as $\mathrm{Si}$ for soil or soluble chlorine for waste incineration ash in urban area. Although the chlorine could have come from sea salt or vegetable burning in rural area. It was found in this study that urban particulates and waste incineration ash source profiles, or soil and coal fly ash source profiles were collinear with the measured species. With lower source contributions, the capability of $\mathrm{CMB}$ receptor to apportion the sources was significantly degraded due to the influence of chemical species with lower precision in both the source and receptor measurements. Modeling results would be deteriorated with the existence of lower precise or inaccurate species, such as $\mathrm{Cd}$. A proposed two-step modeling procedure, which first verifies the measured data from the source contributions of primary (first step) $\mathrm{CMB}$ results and then eliminates those chemical species with lower precision in the second step to obtain better a CMB result, has been demonstrated to effectively improve the capability of CMB model analysis. In addition, the effect of organic and cation species on the model simulation could be an important issues in the future study.

\section{References}

Begum, B.A., Hopke, P.K., Zhao, W., 2005. Source identification of fine particles in Washington, DC, by expanded factor analysis modeling. Environmental Science and Technology 39, 1129-1137.

Bhave, P.V., Pouliot, G.A., Zheng, M., 2007. Diagnostic model evaluation for carbonaceous PM2.5 using organic marker measured in the southeastern US. Environmental Science and Technology 41, 1577-1583.

Christensen, W.F., 2004. Chemical mass balance analysis of air quality data when unknown pollution sources are present. Atmospheric Environment 38, 4305-4317.

Christensen, W.F., Gunst, R.F., 2004. Measurement error models in chemical mass balance analysis of air quality data. Atmospheric Environment 38, 733-744.

Currie, L.A., Gerlach, R.W., Lewis, C.W., Balfour, W.D., Cooper, J.A., Dattner, S.L., DeCesar, R.T., Gordon, G.E., Heisler, S.L., Hopke, P.K., Shah, J.J., Thurston, G.D., Williamson, H.J., 1984. Interlaboratory comparison of source apportionment procedures: results for simulated data sets. Atmospheric Environment 18, 1517-1537.

DeCesar, R.T., Edgerton, S.A., Khalil, M.A.K., Rasmussen, R.A., 1985. Sensitivity analysis of mass balance receptor modeling: methyl chloride as an indicator of wood smoke. Chemosphere 14, 1495-1501. 
Dzubay, T.G., Stevens, R.K., Balfour, W.D., Williamson, H.J., Cooper, J.A., Core, J.E., DeCesar, R.T., Crutcher, E.R., Dattner, S.L., Davis, B.L., Heisler, S.L., Shah, J.J., Hopke, P.K., Johnson, D.L., 1984. Interlaboratory comparison of receptor model results for Houston aerosol. Atmospheric Environment 18, 1555-1566.

Environmental Protection Agency, 2000. EPA-CMB 8.2 User's Manual, EPA publication No. EPA-454/R-00-XXX. Office of Air Quality Planning \& Standards, Research Triangle Park, NC.

Feng, J., Chan, C.K., Fang, M., Hu, M., He, L., Tang, X., 2006. Characteristics of organic matter in PM2.5 in Shanghai. Chemosphere 64, 1393-1400.

Galy, A., Belshaw, N.S., Halicz, L., O'Nions, R.K., 2001. Highprecision measurement of magnesium isotopes by multiplecollector inductively coupled plasma mass spectrometry. International Journal of Mass Spectrometry 208, 89-98.

Gordon, G.E., 1980. Receptor models. Environmental Science and Technology 14, 792-800.

Gordon, G.E., 1988. Receptor models. Environmental Science and Technology 22, 1132-1137.

Gordon, G.E., Zoller, W.H., Kowalczyk, G.S., Rheingrover, S.H., 1981. Composition of Source Components Needed for Aerosol Receptor Models. In: Macias, E.S., Hopke, P.K. (Eds.), Atmospheric Aerosol: Source/Air Quality Relationships, Symposium Series No. 167. American Chemical Society, Washington, DC, pp. 51-74.

Henry, R.C., 1982. Stability Analysis of Receptor Models that Use Least Square Fitting, In Receptor Models Applied to Contemporary Air Pollution Problems, In: Hopke, P.K., Dattner, S.L. (Eds.), Proceedings No. SP-48, Air \& Waste Management Association, Pittsburgh, PA, pp. 141-157.

Hidy, G.M., Friedlander, S.K., 1972. The Nature of the Los Angeles Aerosol, 2nd. IUAPPA Clean Air Congress, Washington, DC.

Hopke, P.K., Dattner, S.L., (Eds.), 1982. Receptor Models Applied to Contemporary Pollution Problems, Proceeding No. SP-48, Air \& Waste Management Association, Pittsburgh, PA.

Hu, W., Hu, B., Jiang, Z., 2006. On-line preconcentration and separation of $\mathrm{Co}, \mathrm{Ni}, \mathrm{Cd}$ via capillary micro-extraction on ordered mesoporous alumina coating and determination by inductively plasma mass spectrometer (ICP-MS). Analytica Chimica Acta 57, 56-62.

Javitz, H.S., Watson, J.G., Robinson, N.F., 1988a. Performance of the chemical mass balance model with simulated local-scale aerosols. Atmospheric Environment 22, 2309-2322.

Javitz, H.S., Watson, J.G., Guertin, J.P., Mueller, P.K., 1988 b. Results of a receptor modeling feasibility study. Journal of Air Pollution Control Association 38, 661-669.

Kawaguchi, H., Tanaka, T., Nakamuram, T., Morishita, M., Mizuike, A., 1987. Matrix effects in inductively coupled plasma mass spectrometry. Analytical Science 3, 305-308.

Kim, E., Hopke, P.K., Pinto, J.P., Wilson, W.E., 2005. Spatial variability of fine particle mass, components, and source continuous during regional air pollution study in St. Louis. Environmental Science and Technology 39, 4172-4179.

Kneip, T.J., Kleinman, M.T., Eisenbud, M., 1972. Relative Contribution of Emission Sources to the Total Airborne Particulates in New York City, 3rd, IUAPPA Clean Air Congress.

Lambkin, D.C., Alloway, B.J., 2000. The problem of arsenic interference in the analysis of soils for cadmium by inductively coupled plasma-optical emission spectrometry. The Science of the Total Environment 256, 77-81.

Larsen, E.H., Sturup, S., 1994. Carbon-enhanced inductively coupled plasma mass spectrometric detection of arsenic and selenium and its application to arsenic speciation. Journal of Analytical Atomic Spectrometry 9, 1099-1105.

Marmur, A., Unal, A., Mulholland, J.A., Russell, A.G., 2005. Optimization-based source apportionment of PM2.5 incorporating gas-to-particle ratios. Environmental Science and Technology 39, 3245-3254.

Olivares, J.A., Houk, R.S., 1986. Suppression of analyte signal by various concomitant salts in inductively coupled plasma mass spectrometry. Analytical Chemistry 58, 20-25.

Robinson, A.L., Subramanian, R., Donahue, N.M., BernardoBricker, A., Rogge, W.F., 2006. Source apportionment of molecular markers and organic aerosol. 2. biomass smoke. Environmental Science and Technology 40, 7811-7819.

Sheppard, B.S., Heitkemper, D.T., Gaston, C.M., 1994. Microwave digestion for the determination of arsenic, cadmium, and lead in seafood products by inductively coupled plasma atomic emission and mass spectrometry. Analyst 119, 1683-1686.

Wang, C.F., Chen, W.H., Yang, M.H., Chiang, P.C., 1995a. Microwave decomposition for airborne particulate matter for the determination of trace elements by inductively coupled plasma mass spectrometry. Analyst 120, 1681-1686.

Wang, C.F., Chang, E.E., Chiang, P.C., Aras, N.K., 1995b. Analytical Procedures on multi-element determinations of airborne particles for receptor model use. Analyst 120, 2521-2528.

Wang, C.F., Huang, M.F., Chang, E.E., Chiang, P.C., $1996 \mathrm{a}$. Assessment of closed vessel digestion methods for elemental determine nation of airborne particulate matter by ICP-AES. Analytical Science 12, 201-207.

Wang, C.F., Yang, J.F., Ke, C.H., 1996b. Multi-element analysis of airborne particulate matter by various spectrometric methods after microwave digestion. Analytica Chimica Acta 320, 207-216.

Wang, C.F., Tu, F.H., Jeng, S.L., 1997. Determination of silicon in airborne particulate matter by UV-visible spectrophotometry. Analytica Chimica Acta 342, 239-245.

Wang, C.F., Chin, C.J., Luo, S.K., Men, L.C., 1999. Determination of chromium in airborne particulate matter by high resolution and laser ablation inductively coupled plasma mass spectrometry. Analytica Chim.ica Acta 389, 257-266.

Ward, T.J., Smith, G.C., 2005. The 2000/2001 Missoula valley PM2.5 chemical mass balance study, including the 2000 wildfire season-seasonal source apportionment. Atmospheric Environment 39, 709-717.

Watson, J.G. (Ed.), 1989. Receptor Models in Air Resources Management, Transactions No. TR-14. Air \& Waste Management Association, Pittsburgh, PA.

Watson, J.G., Robinson, N.F., Chow, J.C., Henry, R.C., Kim, B.M., Pace, T.G., Nguyen, Q., Meyer, E.L., 1990. EPA/DRI Version 7.0 chemical mass balance modeling software. Environmental Software 5, 38-49.

Watson, J.G., Robinson, N.F., Fujita, E.M., Chow, J.C., Pace, T.G., Lewis, C., Coulter, T., 1998. CMB8 Applications and validation protocol for PM2.5 and VOCs, document no. 1808.2D1; Desert Research Institute, Reno, NV.

Winchester, J.W., Nifong, G.D., 1971. Water Pollution in Lake Michigan by Trace Elements from Aerosol Fallout. Water, Air, and Soil Pollution 1, 50-64. 